\title{
Quadrupedal Robots with Stiff and Compliant Actuation
}

\section{Journal Article}

Author(s):

Remy, C. David; Hutter, Marco (D); Hoepflinger, Mark; Bloesch, Michael; Gehring, Christian; Siegwart, Roland

Publication date:

2012-11

Permanent link:

https://doi.org/10.3929/ethz-a-010000217

Rights / license:

In Copyright - Non-Commercial Use Permitted

\section{Originally published in:}

at - Automatisierungstechnik 60(11), https://doi.org/10.1524/auto.2012.1042 


\title{
Quadrupedal Robots with Stiff and Compliant Actuation
}

\author{
Vierbeinige Laufroboter mit weicher und steifer Aktuierung
}

\author{
C. David Remy*, Marco Hutter, Mark Hoepflinger, Michael Bloesch, Christian Gehring, Roland Siegwart, \\ Autonomous Systems Lab, ETH Zürich \\ * Correspondence author: cremy@ethz.ch
}

\begin{abstract}
Summary In the broader context of quadrupedal locomotion, this overview article introduces and compares two platforms that are similar in structure, size, and morphology, yet differ greatly in their concept of actuation. The first, ALoF, is a classically stiff actuated robot that is controlled kinematically, while the second, StarlETH, uses a soft actuation scheme based on highly compliant series elastic actuators. We show how this conceptual difference influences design and control of the robots, compare the hardware of the two systems, and show exemplary their advantages in different applications. $\longrightarrow$ Zusammenfassung Der vorliegende
\end{abstract}

Beitrag vergleicht zwei Laufroboter, die sich in Hinblick auf Struktur, Größe und Morphologie stark ähneln, jedoch im Antriebskonzept klar unterscheiden. Während es sich beim ersten System, ALoF, um einen klassisch angetriebenen Roboter handelt der kinematisch geregelt wird, besitzt der zweite Roboter, StarlETH, Federelemente im Antriebsstrang. Diese ermöglichen eine weiche, kraftgeregelte Aktuierung. Der Beitrag zeigt wie dieser Unterschied Design und Regelung der Roboter beeinflusst, vergleicht die Hardware und erläutert Vor- und Nachteile in verschiedenen Anwendungsfällen.

Keywords Static walking, kinematic motion, force control, series elastic actuation $\gg \quad$ Schlagwörter Statisches Gehen, kinematische Bewegung, Kraft-Regelung, seriell-elastischer Antrieb

\section{Introduction}

It is superfluous to highlight the advantages of legged locomotion for robots that are moving in rough and highly unstructured terrain. While for wheeled systems, mobility is limited by the worst obstacle on a continuous path, legged systems require only a small number of suitable footholds, which can be scattered sparsely throughout the terrain. This makes legs advantageous in situations when a robot is facing gaps, steps, soft ground, or other obstacles. The inherently larger number of degrees of freedom (DoF) can also be used actively to keep the center of gravity $(\mathrm{CoG})$ inside the support area on steep slopes, or to get up after falling down (Fig. 1). That these are not only theoretical benefits becomes immediately obvious when looking at the remarkable locomotion performance of humans and animals that can effortlessly go to places that are completely out of reach for any wheel-based system.
Creating this level of mobility in autonomous robots is a highly desirable goal. Many of the dull, dirty, and dangerous tasks for which we seek to employ autonomous systems must be performed in areas that cannot be

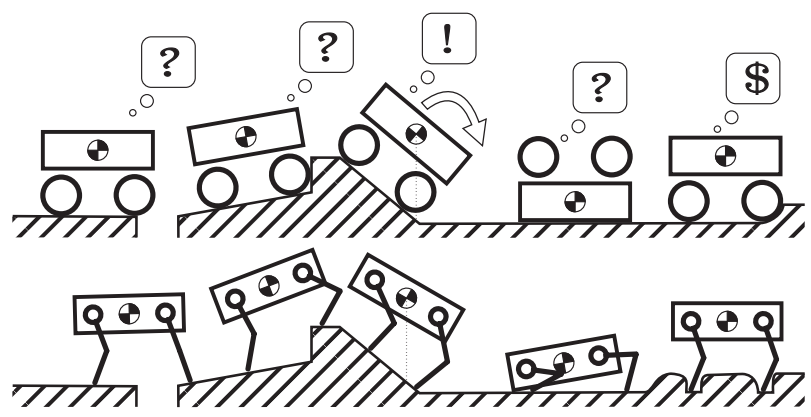

Figure 1 The pictograms illustrate examples of situations in unstructured terrain for which legged locomotion can provide better mobility than wheeled systems. 
reached by wheeled devices. And if we want to create robots that work side-by-side with humans or assist them in their daily lives, it is imperative that they are provided with comparable mobility. Yet, 45 years after the first autonomous walking robot $[1 ; 2]$ and 25 years after Mark Raibert's seminal work on robotic running [3], research on legged locomotion is still facing a considerable performance gap that separates robotic devices from their counterparts in nature. Energy efficiency, locomotion speed, versatility, and robustness, are the most important factors that prevent a wide-spread application of legged locomotion in autonomous systems.

In this context, four legged systems are of a particular research interest for a couple of reasons. First of all, quadrupeds can utilize a rich variety of modes of locomotion, such as walking, trotting, pacing, bounding, or galloping. This variety is largely unstudied in terms of robotic locomotion and can potentially be utilized to improve efficiency and increase the locomotion speed of robotic systems $[4 ; 5]$. Additionally, practical considerations back the choice for quadrupedal systems, especially if they should be employed as research platforms in other areas of mobile robotics, including navigation, path planning [6], or the development of learning algorithms [7]. The possibility for static locomotion on extended support is imperative for such applications; for example, to allow the platform to come to a complete stop. For quadrupeds, the necessary support polygon can be easily created with three or four point feet that span an extended area on the ground. Bipeds, on the other hand, would need extended feet with actuated ankles to maintain static balance. Especially the ankles would thereby greatly increase the mechanical complexity of the system and add more weight to the unsprung mass of the feet. As a result, a heavier foot increases actuator effort for leg swing and foot placement, as well as energetic losses in ground-contact collisions. Additionally, the distributed ground contact of extended feet makes modeling and control very difficult in highly unstructured terrain.

Consequently, as the research-focus in legged locomotion has shifted from Zero Moment Point (ZMP) controlled systems (for which bipedal humanoids are a preferred platform) towards fast, dynamic, and versatile motions, the number of four legged robots has continually increased. Simulations have shown the impressive performance of quadrupeds with abilities to trot, bound, and gallop, while being able to turn and stop quickly [8]; and slowly these abilities are being implemented in practice. Recent robots that are capable of dynamic locomotion include Scamper 1/2 (segmented leg design with stiff actuation) from the lab of Junji Furusho [9], Patrush (segmented leg design with passive ankle springs) from Kimura and his colleagues [10], the KOLT-robot (segmented leg design with parallel actuation in the knee) of Ken Waldron's group at Stanford [11], and Scout I/II (prismatic leg design with a fully passive leg spring) from the Ambulatory Robotics Lab at McGill [12].
The most advanced quadrupedal robot of today is, without doubt, BigDog $[13 ; 14]$ (segmented leg design with hydraulic actuation) from Boston Dynamics, a consistent further development of Marc Raibert's hopping robots $[3 ; 15]$. Its small counterpart, the LittleDog robot also received widespread attention throughout the DARPA Learning Locomotion challenge $[16 ; 17]$ in which a variety of teams demonstrated quadrupedal robotic locomotion in rough terrain. Similar to BigDog with respect to size and actuation, yet much better documented, is HyQ, the hydraulic actuated quadruped from the Italian Institute of Technology [18]. In terms of hardware, the electrically actuated quadruped PQ1-PIRO [19] from the Pohang Institute of Intelligent Robotics is probably closest to the robots presented in this paper.

All these machines can execute a number of different gaits, such as trotting, pacing, and bounding, although some of them are limited to only one or two of these gaits and are not able to perform maneuvers like turning or quickly coming to a stop. Also, none of these machines is yet capable of really fast locomotion or true galloping, although, projects that have locomotion speeds as a primary goal are on their way, for example within DARPA's 'Maximum Mobility and Manipulation (M3) Program'.

In this context, this paper introduces and compares two electrically actuated quadrupedal platforms, ALoF and StarlETH that have both been developed at the Autonomous Systems Lab. The two robots are very similar with respect to their dimensions, weight, and morphology, but they differ greatly in the way they are actuated. ALoF is a classically built stiff robot, in which joints, gearboxes, and motors are connected rigidly. The system is consequently controlled by prescribing kinematic trajectories in joint space. In contrast thereto, StarlETH has series elastic actuation in all its joints that allow high fidelity torque control and enable the usage of advanced control techniques, such as virtual model control [20], operational space control [21], and low gain walking control [6]. In this paper, we will introduce and compare the two platforms, highlight the different control approaches they employ, and seek to quantify the differences in performance and complexity.

\section{Systems Overview}

Both robots that we present are quadrupeds with a total weight in the range of $20 \mathrm{~kg}$ and linear dimensions in the range of half a meter. This means that the platforms are small enough to be handled by one person alone, yet able to carry larger and more sophisticated sensors, as for example stereo cameras, laser range finders, and the like. Each of their legs has three degrees of freedom, allowing for hip abduction/adduction (A/A), hip flexion/extension $(\mathrm{F} / \mathrm{E})$, and knee flexion/extension. In both robots, the first joint (Hip A/A) is aligned along the anterior-posterior axis; a configuration that is commonly referred to as 'mammalian' [22]. The legs are mounted such that the knees of front and back legs are facing each other. We 
chose this configuration because the inner contact forces (i.e., the forces that are created within the ground plane and that are equal in magnitude but opposite in direction) then create the same joint torques in the front and back legs. This is particularly beneficial when the inner contact forces are exploited to reduce the joint torques in the robot, since the resulting torques are distributed more equally throughout the robot. Both systems have point feet that are not actuated. In order to position them freely in three dimensions, at least three active degrees of freedom are necessary per leg. By limiting the number of actual joints to this absolute minimum, complexity is kept low, which increases robustness, modularity, and ease of maintenance of the systems.

\subsection{ALoF}

The first robot, ALoF, the Autonomous Legged Robot on Four legs (Figs. 2 and 3) is built as a classical, kinematically controlled robot with stiff joints. In these, Maxon $R E-2520 \mathrm{~W}$ DC motors are directly connected to the joint axes via planetary gearboxes (reduction 79:1) and a set of bevel-gears (reduction $1: 1$ for Hip A/A), 1.5:1 for Hip $\mathrm{F} / \mathrm{E}$, and $2: 1$ for Knee F/E). In the drive train, elasticities,

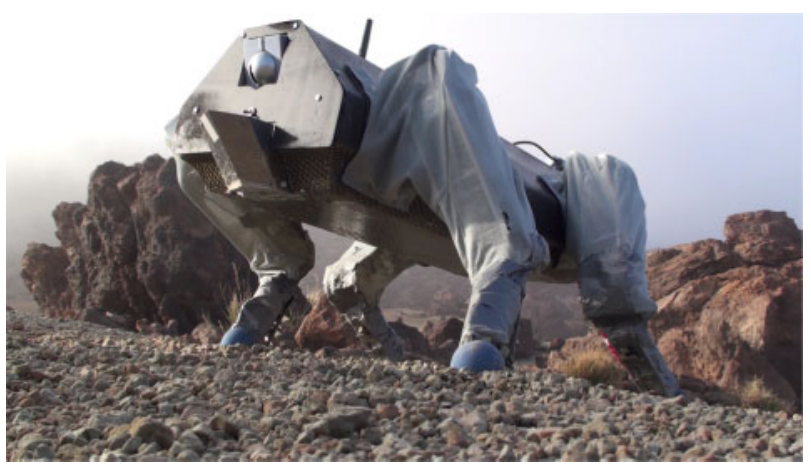

Figure 2 The quadruped robot ALoF. The robot is built with a classically stiffactuation in which joints, gearboxes, and motors are rigidly connected.
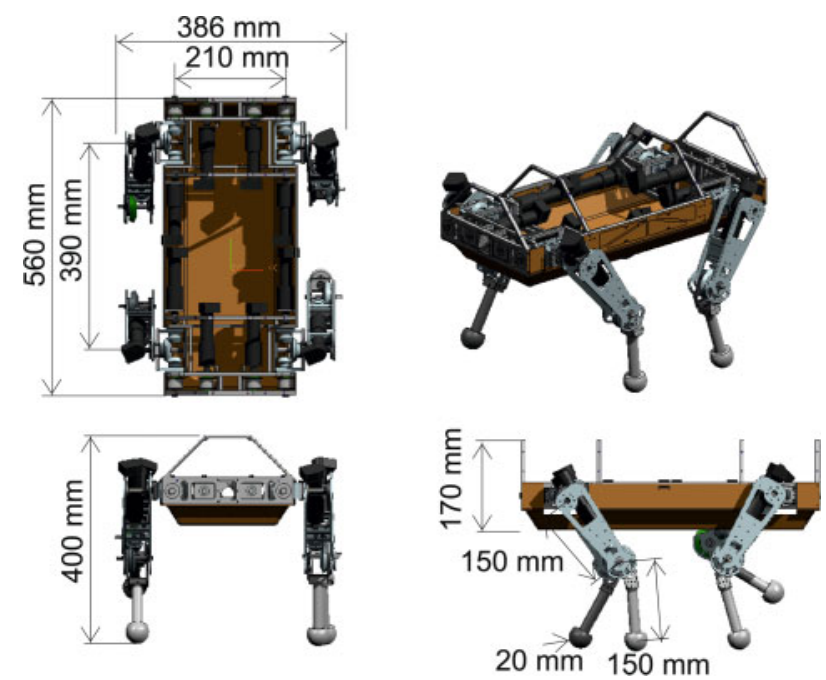

Figure $3 \mathrm{CAD}$ rendering with the dimensions of ALoF. play, and backlash were reduced to the achievable minimum, such that a given motor angle maps uniquely to a specific joint angle. It was hence sufficient to measure only the motor position with an optical encoder and regulate only this quantity. In terms of design, the focus has been put on strong actuation and a large range of motion. The latter was achieved among other design choices by building the hip joint as a universal joint in which hip flexion/extension and hip abduction/adduction are coupled by a differential drive mechanism. This design allows for a greater variation of foot placement and hence provides the robot with the necessary choices for challenging planning tasks. It additionally facilitates haptic exploration of the terrain [7] and enables the execution of alternative gait patterns or recovery maneuvers. This includes, for example, the possibility to perform a crawling motion, which increases the stability of the robot by keeping the center of gravity (COG) low [23]. The entire system and its applications are described in great detail in [24] and [25], to which we would like to refer for further reading.

\subsection{StarlETH}

The Springy Tetrapod with Articulated Robotic Legs, StarlETH (Figs. 4 and 5) is a quadrupedal platform in which all degrees of freedom are driven by series elastic actuators. The leg design for this robot is based on ScarlETH (Series Compliant Articulated Robotic Leg) [26], with an additional degree of freedom for hip abduction/adduction. This leg served as a test-bench to study planar hopping, to design the low-level joint controllers, and to evaluate hardware performance prior to building a full quadrupedal robot. The most crucial components in ScarlETH are the compliant actuators. They are lightweight and arranged in a way that keeps the leg inertia small to reduce impact collision losses and to allow for rapid foot placement. All joints are driven by the same combination of a Maxon EC-4pole-30 $200 \mathrm{~W}$ electric motor and a Harmonic Drive gearing component with a reduction of $100: 1$ (the chain-drive in the knee actuation has an additional reduction of $27: 16$ ). This setup greatly reduces play and back lash in comparison to the drive-train of ALoF. Motor shaft rotations are measured with magnetic incremental encoders. The output shafts of the gearboxes are connected through chain drives and/or steel cable pulley systems with linear compression springs, which are mounted to the adjacent segment. This allows placing all actuators directly at the hip joint and hence minimizes the inertia of the segments. The linear springs show very low mechanical damping, no hysteresis, and are pre-compressed, which makes the system backlash free. High resolution joint encoders (80 000 counts per revolution) allow measuring the joint position and, in combination with the motor encoders, the computation of the spring deflection. This can be directly transformed into joint torque measurements using the known spring characteristic. For initialization 


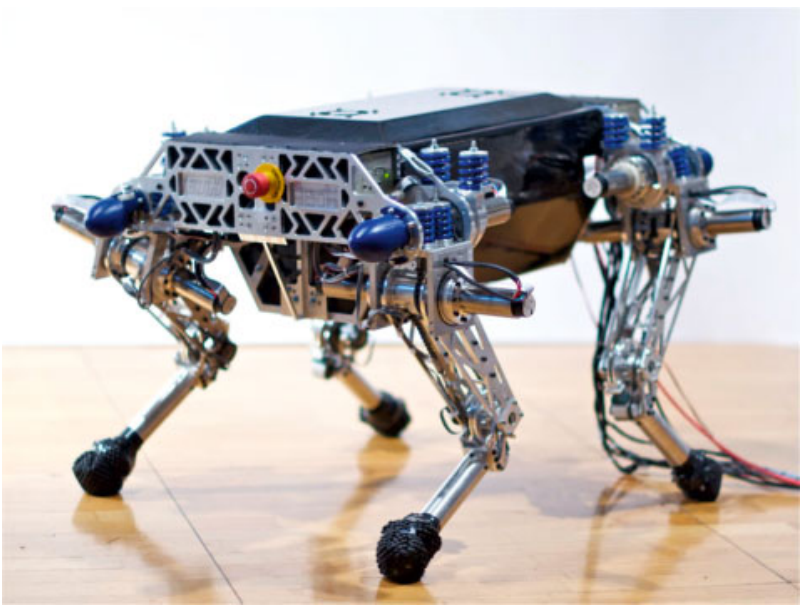

Figure 4 The quadrupedal robotic platform StarlETH. The robot employs series elastic actuation in all 12 joints to allow the exploitation of natural dynamics (photo François Pomerleau).
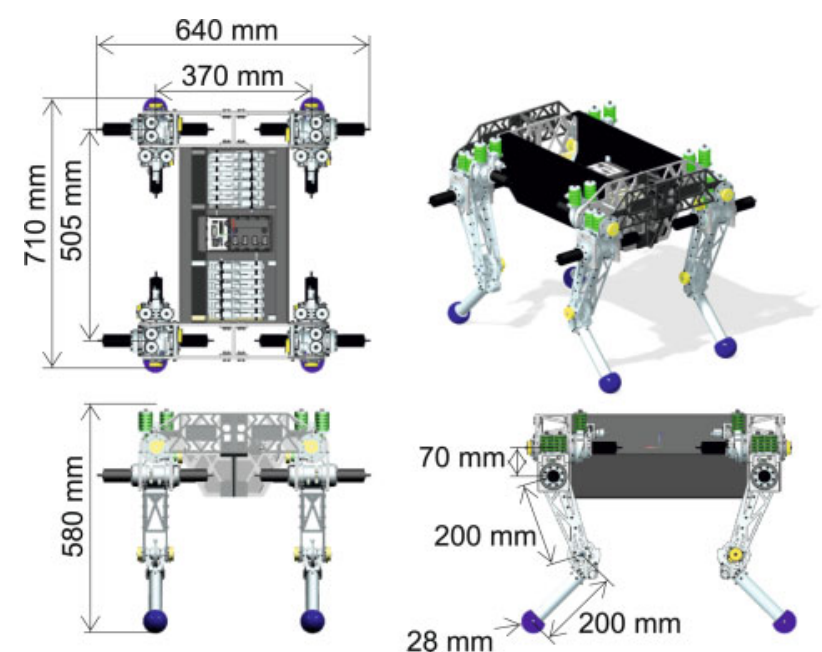

Figure 5 CAD rendering with the dimensions of StarlETH.

and fault detection, all joint angles are additionally measured with potentiometers to obtain an absolute position reference. Motor, gearbox, output shaft, and the springs are included in a highly integrated design that minimizes structural mass. Within the drive train, the chains were deliberately designed as the weakest element in order to protect joints and gears from overload. By using sets of standardized springs with different characteristics, we can alter the stiffness in the system to some degree. Currently we employ a stiffness of $36 \mathrm{Nm} / \mathrm{rad}$ for knee $\mathrm{F} / \mathrm{E}$, $70 \mathrm{Nm} / \mathrm{rad}$ for hip F/E, and $100 \mathrm{Nm} /$ rad for hip A/A. In addition to the elasticity of the springs, other compliances in the actuation chain act in series; for example, the flex spline of the harmonic drives. The overall joint stiffness can thus slightly deviate from the nominal stiffness values of the employed springs. Joint stiffness was consequently identified experimentally with external force-torque sensors. However, it was found to differ only very slightly from the reported spring stiffness values.
In contrast to the traditional use of series elastic actuators (SEA) [27] the springs in StarlETH are highly compliant and allow for larger travel, which has several beneficial properties for legged systems. The compliance protects the gearboxes from impacts at landing and hence allows for highly dynamic maneuvers such as bounding, trotting, or galloping in which such impacts are inevitable. Additionally, the springs can efficiently store and return substantial amounts of energy during stance; for example, up to $30 \mathrm{~J}$ for the knee joint during single legged hopping. Due to the low stiffness, the rate of energy storage and return can be matched with the stride frequency, and the energetic efficiency can be improved. In the example of one-legged hopping about $70 \%$ of the total energy is recovered while only about $30 \%$ need to be provided by the actuators to compensate for impact and damping losses [28]. In a more traditional sense of using SEA's [29], actively controlling the spring deflection [28] or the joint position [26] enables both joint torque as well as joint position control using a cascaded control structure. To improve the position controllability of the knee joint, we included unilateral dampers [26] in the actuator. They ensure fast foot-point tracking during the swing phase without impeding torque control in the stance phase.

\section{Motion Control}

A static walking gait of a quadrupedal robot can be understood as a periodic execution of the lateral footfall sequence [30] left-hind leg (LH), left-front leg (LF), right-hind leg (RH), and right-front leg (RF). On top of this basic pattern, there exist various gait planning algorithms $[31 ; 32]$ that generate the exact contact points as a function of the robot configuration and the surrounding terrain and devise CoG/ZMP trajectories that move the main body forward while ensuring stability of the entire system.

\subsection{Inverse Kinematics}

In stiff position controlled systems such as ALoF, the foot-point and CoG/ZMP trajectories are directly translated into joint space trajectories using inverse kinematics. The resulting desired joint motion is then tracked with high gain position controllers on joint level. This leads to precise trajectory tracking behaviors, but requires that the planned reference trajectories of the CoG as well as of the swing leg precisely match the actual environment. Due to the imperfections in internal and external perception, as well as inaccuracies in the robot model, this kind of controller is not very robust and therefore not well suited for real world applications. In fact, applying such a controller for static walking on terrain with unperceived irregularities may easily lead to loss of stability. In the presence of an unperceived step, for example, the swing leg controller will try to reach a (now erroneous) position goal with all available force and might, in the process, tip over the entire robot [33]. 


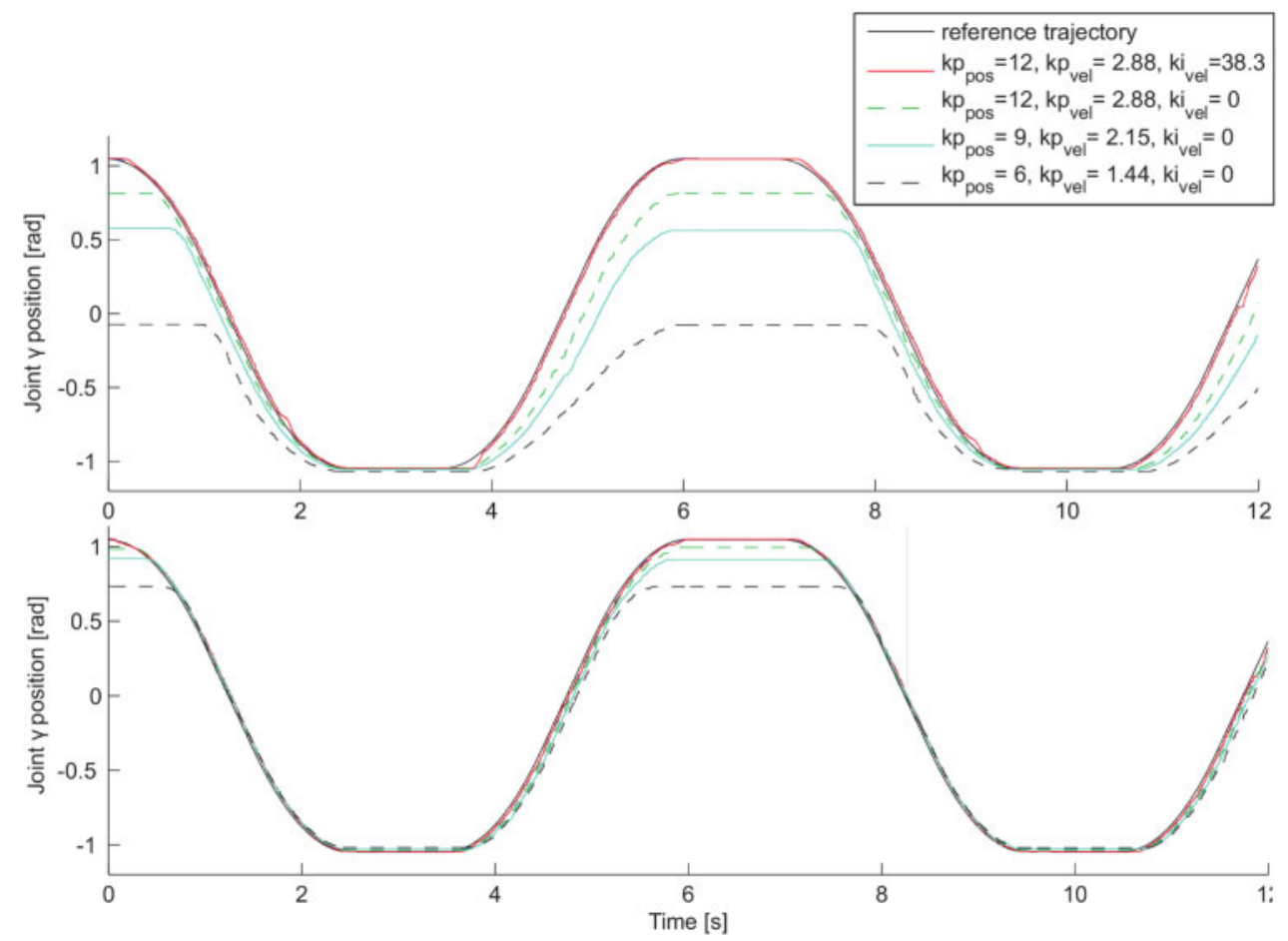

Figure 6 Plots of the knee joint position of a single leg of ALoF under a constant load of about $22 \mathrm{~N}$ in horizontal direction. The trajectories achieved with a position controller without feed-forward (a) and with feed-forward (b) are compared for different controller gains.

One possibility to prevent this is to lower the position control gains at each joint. This low-gain control automatically leads to a compliant behavior that allows for the passive adaptation to irregularities and increases the overall stability. Since this concept works only if we do not employ any integrative controller action, it will also gravely reduce the precision of the executed motion; particularly of the weight bearing joints (i.e., knee and hip F/E of the stance legs). Adding a torque-based feedforward path to the controller architecture to compensate for the effect of gravity can greatly reduce this effect. While we will discuss more sophisticated techniques of doing so below, we want to highlight at this point that even a simple motor and gearbox model [34] and therefore a relatively poor generation of the actual joint torques can already lead to a significant improvement in trajectory tracking performance (Fig. 6). However, it is obvious that the high reflected inertia, the non fully back drivable system, and the highly non-linear friction characteristics in the gearboxes make the application of this method not very practical.

Moving from joint position control (ALoF) to joint torque control (StarlETH) strategies simplifies this process substantially. In the latter, position gains, or joint stiffness, can be arbitrarily chosen and coping with uncertainties in the environment becomes achievable without expensive sensing of the environment.

\subsection{Virtual Model Control}

Instead of changing the dynamics of the system through modulation of the controller gains on joint level, it is ad-

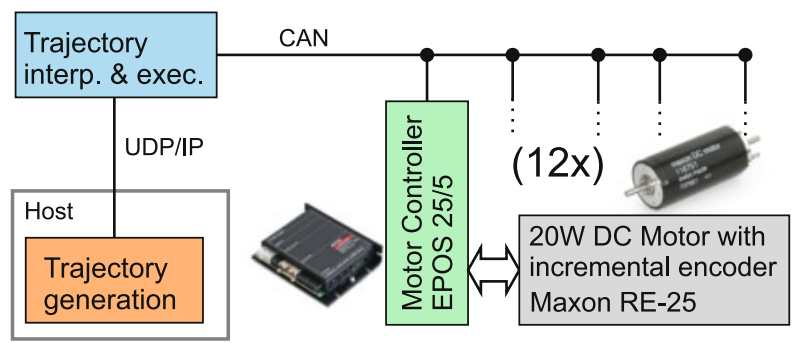

a)

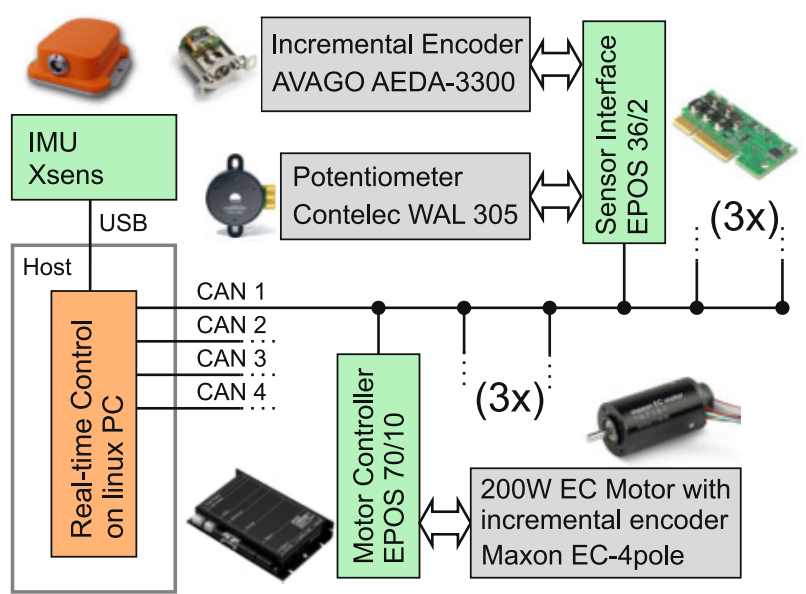

b)

Figure 7 Shown are the electronic hardware components of ALoF (a) and StarlETH (b). In both prototypes, all sensors and motors are connected via CAN bus to a real-time control system. The increased complexity of StarlETH is clearly visible. The extra sensors that measure spring deflections require not only additional sensor boards but also increase data traffic on the bus which makes the use of four individual CAN systems necessary. 
vantageous to directly define a desired dynamic behavior in operational space. As an example, the position $\mathbf{r}_{m b}$ and orientation $\varphi_{m b}$ of the main body should follow a desired trajectory with respect to the world coordinate system given by $\mathbf{r}_{d e s}$ and $\varphi_{\text {des }}$, respectively. For this task, we can define a set of virtual forces $\mathbf{F}_{m b}$ and torques $\mathbf{M}_{m b}$ that correct the current pose according to a desired dynamic behaviour with stiffness $\mathbf{k}_{p}$ and damping $\mathbf{k}_{v}$ :

$\mathbf{F}_{m b}=\mathbf{k}_{p}^{x}\left(\mathbf{r}_{d e s}-\mathbf{r}_{m b}\right)+\mathbf{k}_{d}^{x}\left(\dot{\mathbf{r}}_{d e s}-\dot{\mathbf{r}}_{m b}\right)$

$\mathbf{M}_{m b}=\mathbf{k}_{p}^{\varphi}\left(\varphi_{\text {des }}-\varphi_{m b}\right)+\mathbf{k}_{d}^{\varphi}\left(\dot{\varphi}_{\text {des }}-\dot{\varphi}_{m b}\right)$

In a static approximation, these virtual forces and torques must be balanced by the ground contact forces $\mathbf{F}_{i}^{c}$ of all legs that are in stance $\left(N_{c} \leq 4\right)$ as well as by the gravitational forces $\mathbf{F}_{j}^{g}=-m_{j} \mathbf{g}$ that act on the bodies and segments $\left(N_{b}=13\right)$ :

$\mathbf{F}_{m b}=\sum_{i=1}^{N_{c}} \mathbf{F}_{i}^{c}+\sum_{j=1}^{N_{b}} \mathbf{F}_{j}^{g}$,

$\mathbf{M}_{m b}=\sum_{i=1}^{N_{c}} \mathbf{r}_{i}^{c} \times \mathbf{F}_{i}^{c}+\sum_{j=1}^{N_{b}} \mathbf{r}_{j}^{g} \times \mathbf{F}_{j}^{g}$.

In this, $\mathbf{r}_{i}^{c}$ and $\mathbf{r}_{j}^{g}$ are the vectors from the main body to the $i^{\text {th }}$ contact point and to the CoG of the $j^{\text {th }}$ body, respectively. These equations can be solved for the contact forces by calculating a pseudo inverse that simply minimizes the total contact forces:

$$
\left(\begin{array}{c}
\mathbf{F}_{1}^{c} \\
\mathbf{F}_{2}^{c} \\
\vdots \\
\mathbf{F}_{N_{c}}^{c}
\end{array}\right)=\left[\begin{array}{cccc}
\mathbf{I} & \mathbf{I} & \ldots & \mathbf{I} \\
\tilde{\mathbf{r}}_{1}^{c} & \tilde{\mathbf{r}}_{2}^{c} & \ldots & \tilde{\mathbf{r}}_{N_{c}}^{c}
\end{array}\right]^{+}\left(\begin{array}{c}
\mathbf{F}_{m b}-\sum_{j=1}^{N_{b}} \mathbf{F}_{j}^{g} \\
\mathbf{M}_{m b}-\sum_{j=1}^{N_{b}} \mathbf{r}_{j}^{g} \times \mathbf{F}_{j}^{g}
\end{array}\right) .
$$

(Note: There is a great potential for augmenting this simple least-square optimization. In a terrain with known surface properties, one could for example state the inversion as a constraint optimization problem that minimizes the joint torques while keeping the contact forces inside the available friction cone [35].)

Having the individual ground contact and gravitational forces at hand, a Jacobi-transposed mapping, also known as virtual model control [20], transforms them into joint torques $\mathbf{T}$. It uses the corresponding relative Jacobian of the vector from main body to the foot $i$ given by $\mathbf{J}_{i}=\frac{\partial \mathbf{r}_{i}^{c}}{\partial \mathbf{q}}$, and from the main body to the CoG of body $j$ given by $\mathbf{J}_{j}=\frac{\partial \mathbf{r}_{j}^{g}}{\partial \mathbf{q}}$ :

$\mathbf{T}=\sum_{i=1}^{N} \mathbf{J}_{i}^{T} \mathbf{F}_{i}^{c}+\sum_{j=1}^{N_{b}} \mathbf{J}_{j}^{T} \mathbf{F}_{j}^{g}$

The virtual model control framework allows a very intuitive tuning of the overall behavior in terms of how compliant the main body of the robot reacts to deviations from its predefined trajectory. This results in a very natural reaction against external disturbances, such as pushing the main body, and allows emphasizing stiffness in directions that destabilize the robot while leaving other degrees of freedom more compliant.

\subsection{State Estimation}

Postural controllers, as they are obtained from the virtual model control approach, require fast and precise estimates of the robot's pose. For reasons of mobility and autonomy, this estimation must be solely based on information from on-board sensors and should not utilize external tracking. Especially for dynamic locomotion, this is not a trivial task and the underlying state estimation can quickly become a bottleneck in terms of achievable bandwidth, robustness, and locomotion speed. For optimal performance, we hence designed and implemented an Observability Constrained Extended Kalman Filter [36], that fuses information from the incremental joint encoders with data from an inertial measurement unit. By including the absolute position of all footholds into the filter state, simple and precise model equations can be formulated which accurately capture the uncertainties associated with the intermittent ground contacts. This also enables avoiding unnecessary assumption on the shape of the floor or on the employed gait pattern. Except for the unobservable absolute position and yaw angle (i.e., the rotation around the gravity vector), the resulting filter is able to precisely estimate the full pose of the quadruped robot [37].

\section{Applications}

With their distinct actuator designs, the two robots excel in very different applications and environments. ALoF's primary advantage is its low complexity. In comparison to StarlETH considerably fewer hardware components are required. They come in rugged industrial versions and are packed densely and well protected inside the robot, which allows for a large range of motion.

With these properties ALoF is the designated choice for operation in harsh environments with limited possibilities for control intervention. This was, for example, successfully demonstrated during the Lunar Robotic Challenge of the European Space Agency [23] in which the large range of motion was exploited to perform a static crawling gait which supported the robot stability even in steep terrain with very loose soil [24]. In the challenge, the robot successfully coped with a sandy and dusty environment and even light rain.

The precise execution of kinematically defined motions that can be planned ahead carefully pays off particularly in a number of specific non-locomotion tasks. Figure 8 shows, for example, a maneuver in which the robot starts from a position lying on its back and uses the large range of motion in its joints to flip over and come to rest in its default configuration. This ability 

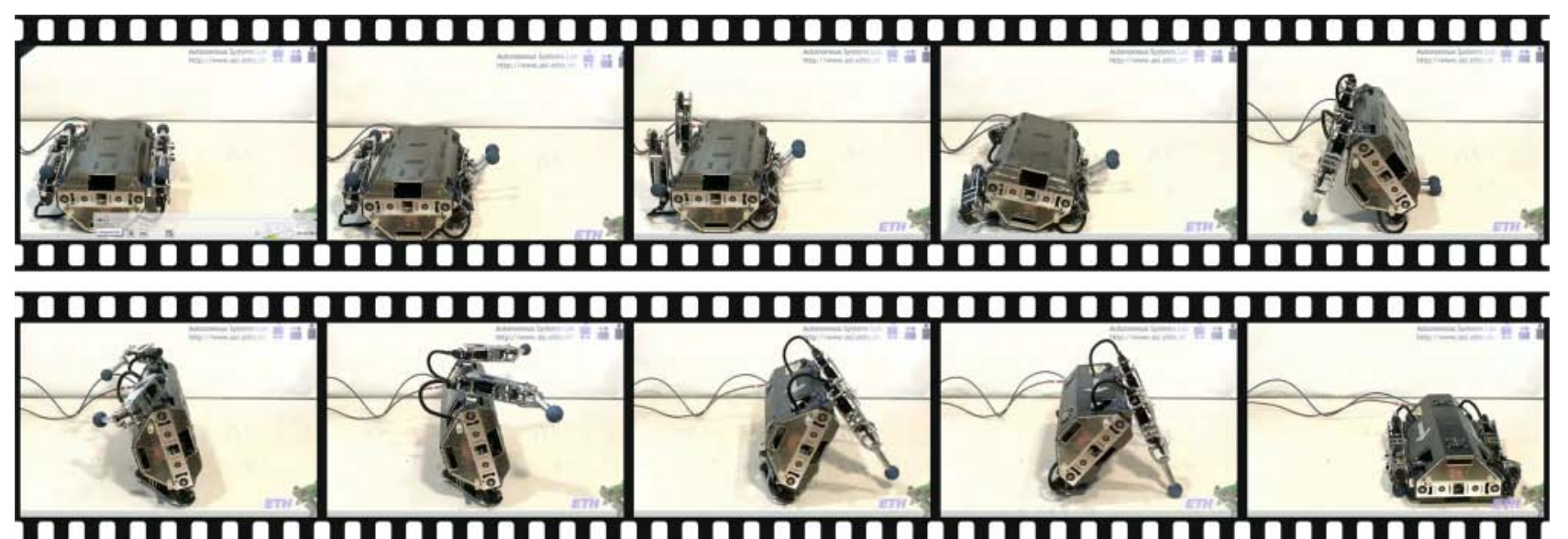

Figure 8 Due to the large range of motion in its joints, ALoF is able to stand up from laying on its back. The ability to perform this task allows the robot to recover from severe falls and creates an intrinsic robustness with respect to locomotion. Throughout the entire maneuver, the robot is supported statically stable by one pair of legs, such that unwanted impacts and collisions are avoided.

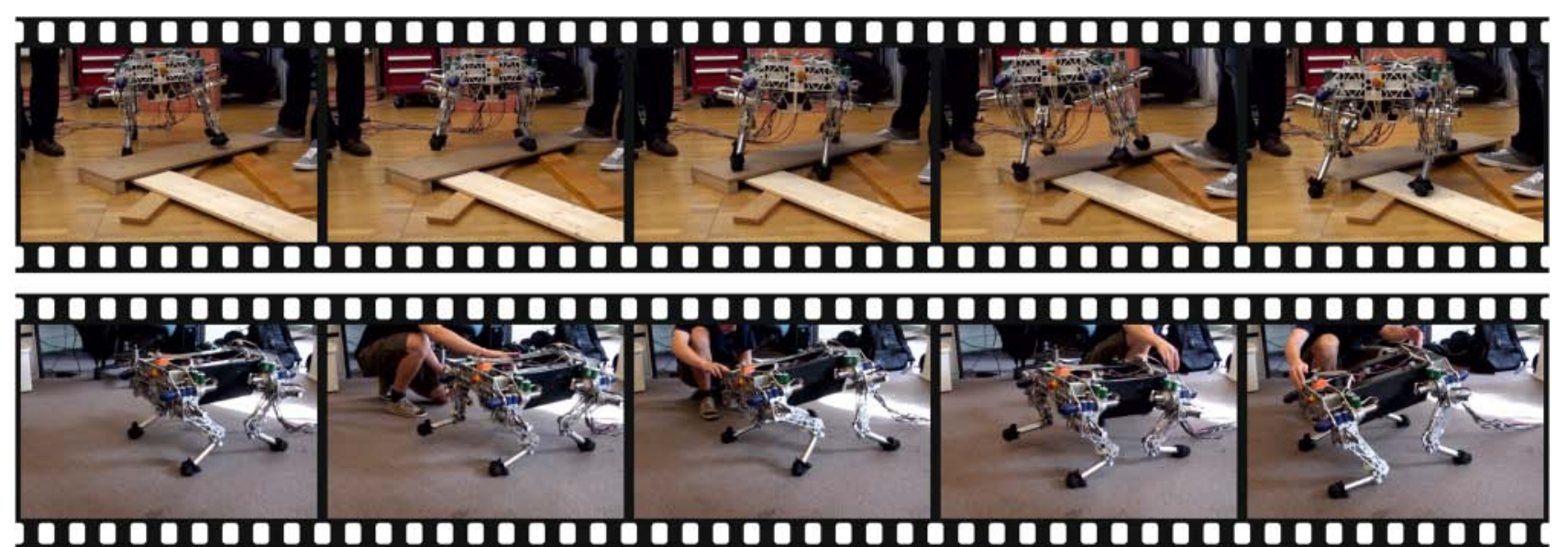

Figure 9 Since StarlETH is fully force controlled, the robot can adapted easily to obstacles and disturbances. The video stills show two different trials in which the robot was commanded to walk straight forward on flat terrain. The obstacles that were added in the top row are not perceived, yet they do not destabilize the robot which automatically adapts to the rough terrain. In the bottom row, a disturbance force is applied to the main body of the robot. Since gravity is compensated through active feed forward, controller gains can be set to very low values and the robot reacts flexible to the disturbances.

adds a very intrinsic robustness to the locomotion capabilities of the robot. Even in the case of the worst possible locomotion disaster, the robot can straighten itself and continue its mission. To the best of our knowledge, it is the first robot able to do so.

The main strength of StarlETH is its adaptability to any kind of irregularity, which results in a greatly increased robustness against disturbances. This can be an unperceived obstacle along the path of the robot or an unknown force acting on its main-body. Figure 9 shows two examples in which the robot is commanded to walk on flat terrain while facing such disturbances. Since the virtual model controller is regulating only the position and orientation of the main body, the kinematic configuration of the robot's joints emerges as a response to the current position of its feet. In contrast to ALoF, for which the position of each foot point must be known precisely and a priory, it is only secondary where the feet are positioned exactly. The robot can hence easily adapt to substantial irregularity in the terrain. The only limiting factors are the range of motion of the legs as well as possible foot-scuffing while swinging a foot over an unperceived obstacle. With conservative joint limits, swing leg trajectories have be devised such that unperceived steps of up to $10 \mathrm{~cm}$ (ca. $20 \%$ of the leg length) were accommodated in hardware experiments. This applies also to dynamical disturbances of the robot's stance; for example if a foot is slipping. Similarly, this holds for disturbances to the main body. When a force is applied to the main body, the robot gives way in accordance to the dynamics of the controlling virtual model while its stance is maintained robustly. Forces of up to $150 \mathrm{~N}$ can be tolerated which can excurse the main body by up to $10 \mathrm{~cm}$. As mentioned before, there are a number of practical advantages that come with the series elastic actuators. While the ability to absorb shocks in unforeseen 
collisions is a simple byproduct of the mechanical design, the increase of efficiency and peak output power through temporary storage of energy require novel controllers that can exploit the natural dynamics of the actuator [5].

\section{Discussion and Conclusions}

For discussion, it would be very tempting to utilize these two nearly identical robots as a unique base for an extensive and quantitative comparison between stiff and soft actuation concepts. However, since the two robots differ in a number of small details and represent a sequential evolution in hardware, one should be very careful with over-interpreting these results. For example, the range of motion of a robot is primarily the consequence of its developer's ability to come up with a tightly integrated design and thus only a secondary consequence of a particular actuator technology. Still, it is obvious that the increased mechanical complexity of StarlETH does not facilitate this job. Also, with the additionally required sensors, the amount of cabling doubles, which further comes in the way of joint motion. So while the limited range of motion of $\operatorname{StarlETH}\left( \pm 30^{\circ}\right.$ for hip A/A, $-40^{\circ} /+80^{\circ}$ for hip $\mathrm{F} / \mathrm{E}$, and $-145^{\circ} /+0^{\circ}$ for knee F/E) is probably not the optimum that is theoretically possible, it indicates how mechanical complexity influences this property. In comparison, ALoF has a range of motion of $\left( \pm 45^{\circ}\right.$ for hip A/A, $\pm 180^{\circ}$ for hip F/E, and $-160^{\circ} /+90^{\circ}$ for knee $\mathrm{F} / \mathrm{E}$ ), which allows the robot to fully contract its legs, rest on its main body, and turn the legs outward and up. With this ability, it can perform maneuvers that are completely out of reach for StarlETH.

The lower complexity of ALoF also applies to control. Kinematic trajectories are pre-computed off-board and can be transmitted to the robot in a coarse temporal resolution $(100 \mathrm{~Hz})$. The necessary data volume is consequently low $(130 \mathrm{kbit} / \mathrm{sec})$ and transmission must not necessarily be done in real time. This relaxes the requirements for the data connection between the robot and a host-PC and allows, for example, the usage of a low-bandwidth wireless link (Fig. 7). The only task that must be accomplished in real-time is the interpolation and execution of these kinematic trajectories, which is done on-board with by a National Instruments singleboardRIO (NI sbRIO-9602 Single-Board RIO Embedded Control with NI-9852 2 Port High-Speed CAN Interface). The link to the host-PC which performs the motion planning and kinematic computations is done via a standard UDP/IP connection.

In contrast, StarlETH must compute the commanded joint torques online and in direct response to the current motion of the robot. Since the output of the controller are torques and not kinematic trajectories, individual joints and even the entire robot can become unstable when they are not under continuous closed-loop control. This defines completely different requirements to the available computational power and the quality of the data links. Consequently, the control algorithms of StarlETH, are
Table 1 Comparison of characteristic properties of the two robots ALoF and StarlETH.

\begin{tabular}{lll}
\hline & ALoF & StarlETH \\
Property & & \\
\hline & 300 & 488.5 \\
Total leg length [mm] & 66 & 68.5 \\
Length of hip segment [mm] & 150 & 200 \\
Length of thigh segment [mm] & 150 & 220 \\
Length of shank segment [mm] & 20 & 55 \\
Foot radius [mm] & 15 & 23 \\
Total mass [kg] & 5 & 25 \\
Max payload for standing up [kg] & 6.15 & 17.5 \\
Weight of actuators [kg] & 0.84 & 2.3 \\
Weight of controllers [kg] & 12 & 49 \\
\# of sensors & $-160 /+90$ & $-145 / 0$ \\
RoM knee F/E [deg] & \pm 180 & $-40 / 80$ \\
RoM hip F/E [deg] & \pm 45 & \pm 30 \\
RoM hip A/A [deg] & $4 / 15$ & $20 / 45$ \\
Torque knee F/E (cont./peak) [Nm] & $3 / 11.3$ & $12 / 24$ \\
Torque hip F/E (cont./peak) [Nm] & $2 / 7.5$ & $12 / 24$ \\
Torque hip A/A (cont./peak) [Nm] & 304 & 350 \\
Max speed knee F/E [deg/s] & 405 & 600 \\
Max speed hip F/E [deg/s] & 608 & 600 \\
Max speed hip A/A [deg/s] & 20 & 200 \\
Motor power rating [W] & & 0 \\
Average play per joint [deg] & & \\
& & \\
\hline
\end{tabular}

executed off-board on a Linux PC and a strictly real time connection with a broad data volume is accomplished via four individual CAN-bus connections. This is imperative, since any time-delay in the connection between the host and the motor-controllers would contribute to the overall controller latency and thus greatly reduce performance and achievable bandwidth.

In terms of hardware, the additional weight for the series elastic actuators (springs, bearings, structural components, as well as sensors and other electronics) sums up to about $1.7 \mathrm{~kg}$ per joint (see Table 1). This is $80 \%$ of the total robot mass. However, this additional mass was more than compensated by the improved mechanical design of StarlETH. In comparison to ALoF, the use of better motors, harmonic drives, and the integration of the drive-train into the mechanical structure, led to an improvement of the power-to-weight ratio from $5.3 \mathrm{~W} / \mathrm{kg}$ to $19.6 \mathrm{~W} / \mathrm{kg}$ (with respect to the total weight of the robots). Consequently the theoretically possible pay-load (in relation to the overall mass) could be increased from $0.33 \mathrm{~kg} / \mathrm{kg}$ to $1.08 \mathrm{~kg} / \mathrm{kg}$. Additionally, pulley-stretch, back lash, and other undesired effects in the drive train (which amount to a play of one degree in ALoF's joints) are almost completely eliminated in StarlETH. Naturally, these improvements are also directly coupled to a substantial increase in the costs for manufacturing and components. In comparison, they roughly doubled from about $35000 \mathrm{CHF}$ to approximately $60000 \mathrm{CHF}$. The increased complexity has also an impact on the energy consumption of the robots. The power that StarlETH draws at rest (i.e., when all motors are disabled) is about $52 \mathrm{~W}$ and thus twice as high as for ALoF (about $27 \mathrm{~W}$ ). This increase can be attributed to 
some degree to the larger amplifiers, but first and foremost to the additional sensors and sensor boards that are needed for the torque-control in StarlETH. During slow static walking, ALoF consumes additionally $29 \mathrm{~W}$ and StarlETH additionally $53 \mathrm{~W}$ to drive their motors. This ratio correlates almost directly with the difference in weight and walking velocity of the two robots, such that the COT for both systems is roughly $10 \mathrm{~J} / \mathrm{Nm}$ for slow static walking. In terms of power consumption in the motors, the series elastic actuation is hence not a direct disadvantage. The potential savings through temporary energy storage in the elastic actuators (as they have already been shown for single leg hopping [26]) have not been exploited in the presented static walking gaits and -with adequate controllers and gaits- might actually improve the overall efficiency.

These properties already show that the current disadvantages of torque controlled robots are not conceptual, but are primarily posing an engineering challenge that will be overcome by better, lighter, and economically more competitive components. Since research in this area is still young, it is not too surprising that adequate offthe-shelf components for control and actuation are not readily available. It is currently not even clear, which system will eventually become the gold standard in terms of actuation. While series elastic actuators have many beneficial properties, intensive and promising research is performed on systems with variable compliance [38] and novel solutions, for example based on MagnetoRehological breaks [39] emerge continuously. Ideally, these solutions provide not only high-fidelity torque outputs, but also reduce the robots mass and inertia, and allow the temporary storage of energy. With these properties it will be possible to build fast, efficient, and versatile robots that are very reliable and allow for save interaction with their users.

\section{Acknowledgements}

This research was supported by the Swiss National Science Foundation through the National Centre of Competence in Research Robotics.

\section{References}

[1] R.B. McGhee. Finite state control of quadruped locomotion. Simulation, 9(3):135-140, 1967.

[2] A. A. Frank. Automatic control systems for legged locomotion. Technical report, University of Southern California, 1968.

[3] M. H. Raibert. Legged robots that balance. The MIT Press series in artificial intelligence. MIT Press, Cambridge, Mass., 1986.

[4] D. F. Hoyt and C. R. Taylor. Gait and the energetics of locomotion in horses. Nature, 292(5820):239-240, 1981.

[5] C. D. Remy, K. W. Buffinton, and R. Y. Siegwart. Energetics of passivity based running with high-compliance series elastic actuation. International Journal of Mechatronics and Manufacturing Systems, 5(2):120-34, 2012.

[6] M. Kalakrishnan, P. Pastor, J. Buchli, M. Mistry, and S. Schaal. Fast, robust quadruped locomotion over challenging terrain. In International Conference on Robotics and Automation, ICRA, pages 2665-2670, 2010.
[7] M. H. Hoepflinger, C. D. Remy, M. Hutter, L. Spinello, and R. Siegwart. Haptic terrain classification for legged robots. In International Conference on Robotics and Automation, ICRA, pages 2828-2833, 2010.

[8] D. Krasny and D Orin. Dynamic maneuvers in a $3 \mathrm{~d}$ galloping quadruped robot (video). In International Conference on Robotics and Automation, ICRA, 2006.

[9] J. Furusho, A. Sano, M. Sakaguchi, and E. Koizumi. Realization of bounce gait in a quadruped robot with articular-joint-type legs. In International Conference on Robotics and Automation, ICRA, volume 1, pages 697-702, 1995.

[10] H. Kimura, S. Akiyama, and K. Sakurama. Realization of dynamic walking and running of the quadruped using neural oscillator. Autonomous Robots, 7(3):247-258, 1999.

[11] J. G. Nichol， S. P. N. Singh， K. J. Waldron, L. R. Palmer III, and D. E. Orin. System design of a quadrupedal galloping machine. International Journal of Robotics Research, pages 1013-1027, 2004.

[12] I. Poulakakis, J. A. Smith, and M. Buehler. Modeling and experiments of untethered quadrupedal running with a bounding gait: The scout ii robot. International Journal of Robotics Research, 24(4):239-256, 2005

[13] M. Buehler, R. Playter, and M. H. Raibert. Robots step outside. Technical report, Boston Dynamics, Sep. 2005.

[14] M. Raibert, K. Blankespoor, G. Nelson, R. Playter, and the BigDog Team. Bigdog, the rough-terrain quadruped robot. In the 17th World Congress The International Federation of Automatic Control (IFAC), 2008.

[15] M. Raibert, M. Chepponis, and H. Brown Jr. Running on four legs as though they were one. Journal of Robotics and Automation, 2(2):70-82, 1986.

[16] M. Kalakrishnan, J. Buchli, P. Pastor, M. Mistry, and S. Schaal. Learning, planning, and control for quadruped locomotion over challenging terrain. The International Journal of Robotics Research, 30(2):236-58, 2010.

[17] K. Byl, A. Shkolnik, S. Prentice, N. Roy, and R. Tedrake. Reliable dynamic motions for a stiff quadruped. In O. Khatib, V. Kumar, and G. Pappas, editors, Experimental Robotics, volume 54 of Springer Tracts in Advanced Robotics, pages 319-328. Springer Berlin/Heidelberg, 2009.

[18] C. Semini. HyQ - Design and Development of a Hydraulically Actuated Quadruped Robot. $\mathrm{PhD}$ thesis, University of Genoa, Italy and Italian Institute of Technology (IIT), 2010.

[19] M. Won, T. Kang, and W. Chung. Gait planning for quadruped robot based on dynamic stability: landing accordance ratio. Intelligent Service Robotics, 2:105-112, 2009. 10.1007/s11370-009-0038-7.

[20] J. Pratt, C. M. Chew, A. Torres, P. Dilworth, and G. Pratt. Virtual model control: An intuitive approach for bipedal locomotion. International Journal of Robotics Research, 20(2):129-43, 2001.

[21] L. Sentis. Compliant Control of Whole-Body Multi-Contact Behaviors in Humanoid Robots, chapter 2, pages 29-66. Springer Global Editorial, 2009.

[22] G. Genta. Chapter 6.1, walking machines. In Introduction to the Mechanics of Space Robots, pages 381-409. Springer, 2012.

[23] F. A. W. Belo, A. Birk, C. Brunskill, F. Kirchner, V. Lappas, C. D. Remy, S. Roccella, C. Rossi, A. Tikanmäki, and G. Visentin. The esa lunar robotics challenge: Simulating operations at the lunar south pole. Journal of Field Robotics, 29(4):601-26, 2012.

[24] C. D. Remy, O. Baur, M. Latta, A. Lauber, M. Hutter, M. H. Hoepflinger, C. Pradalier, and R. Siegwart. Walking and crawling with alof - a robot for autonomous locomotion on four legs. Industrial Robot: An International Journal, 38(3):564-268, 2011.

[25] M. H. Hoepflinger, C. D. Remy, M. Hutter, and R. Siegwart. The quadruped alof and a step towards real world haptic terrain classification. In Mechatronics 2010, pages 173-180, 2010.

[26] M. Hutter, C. D. Remy, M. H. Hoepflinger, and R. Siegwart. High compliant series elastic actuation for the robotic leg scarleth. In International Conference on CLimbing And WAlking Robots, CLAWAR, 2011. 
[27] G. Pratt and M. Williamson. Series elastic actuators. In International Conference on Intelligent RObots and Systems, IROS, volume 1, pages 399-06, 1995.

[28] M. Hutter, C. D. Remy, M. H. Hoepflinger, and R. Siegwart. ScarlETH: Design and control of a planarrunning robot. In International Conference on Intelligent RObots and Systems, IROS, pages 562-67, 2011.

[29] J. Pratt, B. Krupp, and C. Morse. Series elastic actuators for high fidelity force control. Industrial Robot, 29(3):234-41, 2002.

[30] M. Hildebrand. The adaptive significance of tetrapod gait selection. Integrative and Comparative Biology, 20(1):255-267, 1980.

[31] J.Z. Kolter and A.Y. Ng. The stanford littledog: A learning and rapid replanning approach to quadruped locomotion. The International Journal of Robotics Research, 30(2):150-174, 2011.

[32] M. Zucker, N. Ratliff, M. Stolle, J. Chestnutt, J. A. Bagnell, C. G. Atkeson, and J. Kuffner. Optimization and learning for rough terrain legged locomotion. The International Journal of Robotics Research, 30(2):175-191, 2011.

[33] J. Buchli, M. Kalakrishnan, M. Mistry, P. Pastor, and S. Schaal. Compliant quadruped locomotion over rough terrain. In International Conference on Intelligent RObots and Systems, IROS, pages 814-820, 2009.

[34] M. Brändle. From little-dog to alof. Master thesis, ETH Zurich, 2010.

[35] M. Bloesch. State estimation and robust control for a quadruped robot. Master thesis, ETH Zurich, 2011.

[36] G. P. Huang, A. I. Mourikis, and S. I. Roumeliotis. Observabilitybased rules for designing consistent ekf slam estimators. International Journal of Robotics Research, 29:502-528, Apr. 2010.

[37] M. Bloesch, M. Hutter, M. H. Hoepflinger, C. D. Remy, C. Gehring, and R. Siegwart. State estimation for legged robots - consistent fusion of leg kinematics and imu. In Proceedings of Robotics: Science and Systems, 2012.

[38] L. C. Visser, R. Carloni, R. Unal, and S. Stramigioli. Modeling and design of energy efficient variable stiffness actuators. In International Conference on Robotics and Automation, ICRA, pages 3273 -3278 , may 2010

[39] P. Fauteux, M. Lauria, B. Heintz, and F. Michaud. Dual differential rheological actuator for high performance physical robotic interaction. IEEE Transactions on Robotics, 26:607-618, 2010.
Received: July 3, 2012

Prof. Dr. C. David Remy is Assistant Professor at the Department of Mechanical Engineering of the University of Michigan. Research interest: Exploitation of natural dynamics in legged systems.

Address: University of Michigan, Department of Mechanical Engineering, Ann Arbor, MI-48109, Tel.: +1-(734)-764-8797,

e-mail: cdremy@umich.edu

MSc Marco Hutter is a PhD student at the Autonomous Systems Lab, ETH Zurich. Research interest: Design and control of compliant walking and runnig machines.

Address: ETH Zurich, Autonomous Systems Lab, CH-8092 Zürich, Tel.: +41-(0)44-632-7417, e-mail: mahutter@ethz.ch

MSc Mark H. Hoepflinger is a PhD student at the Autonomous Systems Lab, ETH Zurich. Research interest: Robust legged locomotion in rough terrain.

Address: ETH Zurich, Autonomous Systems Lab, CH-8092 Zürich, Tel.: +41-(0)44-632-2145, e-mail: markho@ethz.ch

MSc Michael Bloesch is a PhD student at the Autonomous Systems Lab, ETH Zurich. Research interest: Stochastic state estimation and mapping, control design for legged locomotion.

Address: ETH Zurich, Autonomous Systems Lab, CH-8092 Zürich Tel.: +41-(0)44-632-9871, e-mail: bloeschm@ethz.ch

MSc Christian Gehring is a PhD student at the Autonomous Systems Lab, ETH Zurich. Research interest: Agile and robust locomotion with compliant quadrupeds.

Address: ETH Zurich, Autonomous Systems Lab, CH-8092 Zürich, Tel.: +41-(0)44-632-9871, e-mail: gehrinch@ethz.ch

Prof. Dr. Roland Siegwart is a Full Professor for Autonomous Systems at ETH Zurich and the Vice President Research and Corporate Relations. Research interests: Design and control of systems operating in complex and highly dynamical environments.

Address: ETH Zurich, Autonomous Systems Lab, CH-8092 Zürich Tel.: +41-(0)44-632-2358, e-mail: rsiegwart@ethz.ch 